\title{
A Shifted Jacobi-Gauss Collocation Scheme for Solving Fractional Neutral Functional-Differential Equations
}

\author{
A. H. Bhrawy ${ }^{1,2}$ and M. A. Alghamdi ${ }^{1}$ \\ ${ }^{1}$ Department of Mathematics, Faculty of Science, King Abdulaziz University, Jeddah 21589, Saudi Arabia \\ ${ }^{2}$ Department of Mathematics, Faculty of Science, Beni-Suef University, Beni-Suef 62511, Egypt \\ Correspondence should be addressed to A. H. Bhrawy; alibhrawy@yahoo.co.uk
}

Received 4 March 2014; Revised 29 March 2014; Accepted 29 March 2014; Published 15 April 2014

Academic Editor: Xiao-Jun Yang

Copyright (c) 2014 A. H. Bhrawy and M. A. Alghamdi. This is an open access article distributed under the Creative Commons Attribution License, which permits unrestricted use, distribution, and reproduction in any medium, provided the original work is properly cited.

\begin{abstract}
The shifted Jacobi-Gauss collocation (SJGC) scheme is proposed and implemented to solve the fractional neutral functionaldifferential equations with proportional delays. The technique we have proposed is based upon shifted Jacobi polynomials with the Gauss quadrature integration technique. The main advantage of the shifted Jacobi-Gauss scheme is to reduce solving the generalized fractional neutral functional-differential equations to a system of algebraic equations in the unknown expansion. Reasonable numerical results are achieved by choosing few shifted Jacobi-Gauss collocation nodes. Numerical results demonstrate the accuracy, and versatility of the proposed algorithm.
\end{abstract}

\section{Introduction}

Fractional differential equations (FDEs) have drawn the interest of many researchers in recent years [1-6], due to their useful applications in many fields of science. In fact, we may observe several applications in electrochemistry, viscoelasticity, electromagnetic, control, plasma physics, porous media, fluctuating environments, dynamical processes, and so on. In consequence, fractional differential equations are gaining much attention from the researchers. For some recent developments on this subject, see [7-15].

In the last decade or so, comprehensive research has been accomplished on the development of numerical algorithms which are numerically stable for both linear and nonlinear FDEs. Tripathi et al. [16] presented a new operational matrix of hat functions to solve linear FDEs. The spectral tau method was proposed in [17] to achieve an accurate solution of linear and nonlinear FDEs subject to multipoint conditions. In [18], the author proposed Bernstein polynomial to design a numerical algorithm for fractional Riccati equations. The authors of [19] investigated the spline collocation method for approximating the solution of nonlinear FDEs. Furthermore, the author of [20] transformed the time-dependent space
FDE with variable coefficients into a system of ordinary differential equations, which is then solved by a standard numerical method. Baleanu et al. [21] developed the generalized Laguerre spectral tau and collocation approximations to solve FDEs on the half line. In [22], Ma and Huang developed spectral collocation method for solving linear fractional integrodifferential equations. Yang and Huang [23] analyzed and developed the Jacobi collocation scheme for pantograph integrodifferential equations with fractional orders in finite interval. In [24] Yin et al. proposed a new fractional-order Legendre function with spectral method to solve partial FDEs; based on the operational matrix of these functions, the same authors developed their approach in combination with variational iteration formula to solve a class of FDEs; see [25]. More recently, the Jacobi Galerkin method was extended in [26] to solve stochastic FDEs.

Polynomial approximations can be quite useful for expressing the solution of a differential equation. One such approach would be the spectral methods. An advantage of a spectral collocation method is that it gives high accurate solutions with relatively fewer spatial grid nodes when compared with other numerical techniques. In [27], the Jacobi rational collocation scheme was proposed and developed to 
solve generalized pantograph equations. In [28], the authors extended the application of Jacobi-Gauss-Lobatto collocation approximation to solve $(1+1)$ nonlinear Schrödinger equations. Also, the generalized Laguerre-Legendre collocation method has been successfully applied to initial-boundary value problems [29]. In [30], approximate solutions of nonlinear Klein-Gordon and Sine-Gordon equations were provided using the Chebyshev tau meshless scheme. For some recent developments on spectral methods, see [31-34].

Neutral functional-differential equations play an important role in the mathematical modeling of several phenomena. It is well known that most of delay differential equations cannot be solved exactly. Therefore, numerical methods would be presented and developed to get approximate solutions of these equations. In this direction, Ishiwata and Muroya [35] applied the rational approximation scheme for solving a class of delay differential equations. In [36], Chen and Wang implemented the variational iteration scheme to obtain an analytical solution of the neutral functional-differential equation. Very recently, Heydari et al. [37] proposed a new numerical algorithm based on the operational matrix formulation of Chebyshev cardinal functions for solving delay differential equations arising in electrodynamics. In this paper we propose a numerical solution for a new class of delay differential equations, namely, fractional neutral functional-differential equations (FNFDEs) with proportional delay.

The main aim of this paper is to design a suitable way to approximate a new class of functional-differential equations with fractional orders on the interval $(0, L)$ using spectral collocation method. The spectral shifted Jacobi-Gauss collocation (SJGC) approximation is proposed to obtain the numerical solution $u_{N}(t)$. The SJGC approximation, which is more reliable, is employed to obtain approximate solution of FNFDEs with leading fractional order $\theta(m-1<\theta<m)$ and $m$ initial conditions. We choose the $(N-m+1)$ nodes of the shifted Jacobi-Gauss interpolation on $(0, L)$ as suitable collocation nodes. The Legendre and Chebyshev collocation approximations can be obtained as special cases from our general approach. Finally, the validity and effectiveness of the method are demonstrated by solving two numerical examples. Numerical examples are presented in the form of tables and graphs to make comparisons with the results obtained by other methods and with the exact solutions more easier.

In the next section, we present an overview of shifted Jacobi polynomials and fractional calculus needed hereafter. Section 3 is devoted to present and implement the collocation scheme for solving FNFDEs with proportional delay using Jacobi polynomials. In Section 4, we introduce two numerical examples demonstrating the high accuracy and efficiency of the present numerical algorithm.

\section{Preliminaries}

Here, we state some preliminaries of fractional calculus [38] and some relevant properties of Jacobi polynomials. The most commonly used definition of fractional integral is the Riemann-Liouville operator.
Definition 1. The Riemann-Liouville fractional integral operator of $\operatorname{order} \theta(\theta>0)$ is defined as

$$
\begin{gathered}
J^{\theta} f(t)=\frac{1}{\Gamma(\theta)} \int_{0}^{t}(t-s)^{\theta-1} f(s) d s, \quad \theta>0, t>0, \\
J^{0} f(t)=f(t) .
\end{gathered}
$$

Definition 2. The Caputo fractional derivatives of order $\theta$ are defined as

$$
\begin{aligned}
D^{\theta} f(t) & =J^{m-\theta} D^{m} f(t) \\
= & \frac{1}{\Gamma(m-\theta)} \int_{0}^{t}(t-s)^{m-\theta-1} \frac{d^{m}}{d s^{m}} f(s) d s, \\
& \quad m-1<\theta<m, t>0,
\end{aligned}
$$

where $D^{m}$ is the classical differential operator of order $m$.

Also

$$
D^{\theta} C=0, \quad(C \text { is aconstant }),
$$

$$
\begin{aligned}
& D^{\theta} t^{\mu} \\
& \quad=\left\{\begin{array}{l}
0, \\
\quad \text { for } \mu \in N_{0}, \mu<\lceil\theta\rceil, \\
\frac{\Gamma(\mu+1)}{\Gamma(\mu+1-\theta)} t^{\mu-\theta}, \\
\quad \text { for } \mu \in N_{0}, \mu \geq\lceil\theta\rceil \text { or } \mu \notin N, \mu>\lfloor\theta\rfloor,
\end{array}\right.
\end{aligned}
$$

where $N=\{1,2, \ldots\}$ and $N_{0}=\{0,1,2, \ldots\}$, while $\lceil\theta\rceil$ and $\lceil\theta\rceil$ are the floor and ceiling functions, respectively,

The Caputo's fractional differentiation is a linear operation, similar to the integer-order differentiation

$$
D^{\theta}(\lambda f(t)+\eta g(t))=\lambda D^{\theta} f(t)+\eta D^{\theta} g(t),
$$

where $\lambda$ and $\eta$ are constants.

Let $\nu>-1, \mu>-1$ and let $P_{k}^{(\nu, \mu)}(t)$ be the Jacobi polynomial of degree $k$; then we get

$$
\begin{gathered}
P_{k}^{(\nu, \mu)}(-t)=(-1)^{k} P_{k}^{(\nu, \mu)}(t), \\
P_{k}^{(\nu, \mu)}(-1)=\frac{(-1)^{k} \Gamma(k+\mu+1)}{k ! \Gamma(\mu+1)}, \\
P_{k}^{(\nu, \mu)}(1)=\frac{\Gamma(k+\nu+1)}{k ! \Gamma(\nu+1)} .
\end{gathered}
$$

Besides,

$$
D^{m} P_{k}^{(v, \mu)}(t)=2^{-m} \frac{\Gamma(m+k+v+\mu+1)}{\Gamma(k+v+\mu+1)} P_{k-m}^{(\nu+m, \mu+m)}(t) .
$$

Let $w^{(\nu, \mu)}(t)=(1-t)^{\nu}(1+t)^{\mu}$; then we define the weighted space $L_{w^{(\nu, \mu)}}^{2}[-1,1]$ as usual, equipped with the following inner product and norm:

$$
\begin{gathered}
(u, v)_{w^{(\nu, \mu)}}=\int_{-1}^{1} u(t) v(t) w^{(v, \mu)}(t) d t \\
\|v\|_{w^{(v, \mu)}}=(v, v)_{w^{(v, \mu)}}^{1 / 2} .
\end{gathered}
$$


The set of Jacobi polynomials forms a complete $L_{w^{\nu, \mu}}^{2}[-1,1]$ orthogonal system, and

$$
\begin{aligned}
\left\|P_{k}^{(\nu, \mu)}\right\|_{w^{(\nu, \mu)}}^{2} & =h_{k}^{(\nu, \mu)} \\
& =\frac{2^{\nu+\mu+1} \Gamma(k+\nu+1) \Gamma(k+\mu+1)}{(2 k+\nu+\mu+1) \Gamma(k+1) \Gamma(k+\nu+\mu+1)} .
\end{aligned}
$$

Let us define the shifted Jacobi polynomial of degree $k$ by $P_{L, k}^{(\nu, \mu)}(t)=P_{k}^{(\nu, \mu)}((2 t / L)-1), L>0$, and thanks to (6) and (7), yield

$$
\begin{aligned}
& D^{q} P_{L, k}^{(\nu, \mu)}(0)=\frac{(-1)^{k-q} \Gamma(k+\mu+1)(k+v+\mu+1)_{q}}{L^{q} \Gamma(k-q+1) \Gamma(q+\mu+1)}, \\
& D^{m} P_{L, k}^{(\nu, \mu)}(t)=\frac{\Gamma(m+k+v+\mu+1)}{L^{m} \Gamma(k+v+\mu+1)} P_{L, k-m}^{(\nu+m, \mu+m)}(t) .
\end{aligned}
$$

The shifted Jacobi polynomials of degree $i$ on the interval $[0, L]$ are given by

$$
\begin{aligned}
P_{L, i}^{(\nu, \mu)}(t) & \\
=\sum_{k=0}^{i}(-1)^{i-k}( & \left(\Gamma(i+\mu+1) \Gamma(i+k+\nu+\mu+1) t^{k}\right) \\
& \times(\Gamma(k+\mu+1) \Gamma(i+\nu+\mu+1) \\
& \left.\left.\times(i-k) ! k ! L^{k}\right)^{-1}\right) \quad i=0,1, \ldots
\end{aligned}
$$

Since the analytic form of $P_{L, i}^{(v, \mu)}(t)$ is given by (12), with the use of (4), (5), and (12), we obtain

$$
\begin{aligned}
& D^{\theta} P_{L, i}^{(\nu, \mu)}(t) \\
& =\sum_{k=0}^{i}(-1)^{i-k}((\Gamma(i+\mu+1) \Gamma(i+k+\nu+\mu+1)) \\
& \times(\Gamma(k+\mu+1) \Gamma(i+\nu+\mu+1) \\
& \left.\left.\times(i-k) ! k ! L^{k}\right)^{-1}\right) D^{\theta} t^{k} \\
& =\sum_{k=\lceil\theta\rceil}^{i}(-1)^{i-k}((\Gamma(i+\mu+1) \Gamma(i+k+\nu+\mu+1)) \\
& \times(\Gamma(k+\mu+1) \Gamma(i+\nu+\mu+1) \\
& \left.\left.\times(i-k) ! \Gamma(k-\theta+1) L^{k}\right)^{-1}\right) t^{k-\theta}, \\
& i=\lceil\theta\rceil, \ldots, N \text {. }
\end{aligned}
$$

Now, approximate $t^{k-\theta}$ by $N+1$ terms of shifted Jacobi series; we have

$$
t^{k-\theta}=\sum_{j=0}^{N} b_{j} P_{L, j}^{(\nu, \mu)}(t)
$$

where $b_{j}$ is given from (18) with $u(x)=t^{k-\theta}$, and

$$
\begin{aligned}
& b_{j}=\frac{L^{\nu+\mu-\theta+1}}{h_{j}^{(v, \mu)} \Gamma(j+\nu+\mu+1)} \\
& \times \sum_{\ell=0}^{j}\left(\left((-1)^{j-\ell} \Gamma(j+\ell+\nu+\mu+1) \Gamma(\nu+1)\right.\right. \\
&\times \Gamma(\ell+k-\theta+\nu+\ell+1)) \\
& \times(\Gamma(\ell+\mu+1)(j-\ell) !(\ell) ! \\
&\left.\quad \times \Gamma(\ell+k-\theta+v+\ell+2 \Gamma))^{-1}\right) .
\end{aligned}
$$

Employing (13)-(15) we get

$$
D^{\theta} P_{L, i}^{(\nu, \mu)}(t)=\sum_{j=0}^{N} S_{\theta}(i, j) P_{L, j}^{(\nu, \mu)}(t), \quad i=\lceil\theta\rceil, \ldots, N
$$

where $S_{\theta}(i, j)=\sum_{k=\lceil\theta\rceil}^{i} \vartheta_{i j k}$, and

$$
\begin{aligned}
\vartheta_{i j k}=( & (-1)^{i-k} L^{\nu+\mu-\theta+1} \Gamma(j+\mu+1) \\
& \times \Gamma(i+\mu+1) \Gamma(i+k+v+\mu+1)) \\
& \times\left(h_{j}^{(\nu, \mu)} \Gamma(j+v+\mu+1) \Gamma(k+\mu+1)\right. \\
& \times \Gamma(i+v+\mu+1) \Gamma(k-\theta+1)(i-k) !)^{-1} \\
\times & \sum_{\ell=0}^{j}\left(\left((-1)^{j-\ell} \Gamma(j+\ell+v+\mu+1) \Gamma(\nu+1)\right.\right. \\
\times & \times(\ell+k-\theta+v+\ell+1)) \\
& \times(\ell+\mu+1)(j-\ell) !(\ell) !
\end{aligned}
$$

A function $u(t) \in L_{w^{(v, \mu)}}^{2}(0, L)$ may be expressed in terms of shifted Jacobi polynomials as

$$
\begin{gathered}
u(t)=\sum_{j=0}^{\infty} a_{j} P_{L, j}^{(\nu, \mu)}(t), \\
a_{j}=\frac{1}{h_{k}^{(v, \mu)}} \int_{0}^{L} u(t) P_{L, j}^{(\nu, \mu)}(t) w^{(\nu, \mu)}(t) d t, \quad j=0,1,2, \ldots
\end{gathered}
$$

In practice, only the first $(N+1)$ terms shifted Jacobi polynomials are considered. Then we have

$$
u_{N}(t)=\sum_{j=0}^{N} a_{j} P_{L, j}^{(v, \mu)}(t)
$$


Next, let $w_{L}^{(v, \mu)}(t)=(L-t)^{v} t^{\mu}$; then we define the weighted space $L_{w_{L}^{(\gamma, \mu)}}^{2}[0, L]$ in the usual way, with the following inner product and norm:

$$
\begin{gathered}
(u, v)_{w_{L}^{(\nu, \mu)}}=\int_{0}^{L} u(t) v(t) w_{L}^{(\nu, \mu)}(t) d t, \\
\|v\|_{w_{L}^{(\nu, \mu)}}=(v, v)_{w_{L}^{1 / \mu, \mu)}}^{1 / 2} .
\end{gathered}
$$

The set of shifted Jacobi polynomials forms a complete $L_{w_{L}^{(\gamma, \mu)}}^{2}[0, L]$-orthogonal system. Moreover, and due to (9), we have

$$
\left\|P_{L, k}^{(v, \mu)}\right\|_{w_{L}^{(\gamma, \mu)}}^{2}=\left(\frac{L}{2}\right)^{\nu+\mu+1} h_{k}^{(v, \mu)}=h_{L, k}^{(v, \mu)} .
$$

\section{Shifted Jacobi Collocation Approximation for FNFDEs}

In this section, we propose the shifted Jacobi collocation method with the Jacobi-Gauss quadrature nodes to solve numerically the following FNFDEs with proportional delay:

$$
\begin{aligned}
D^{\theta} & \left(u(t)+a(t) u\left(p_{m} t\right)\right) \\
& =\mu u(t)+\sum_{n=0}^{m-1} b_{n}(t) D^{\gamma_{n}} u\left(p_{n} t\right)+f(t), \quad t \geq 0,
\end{aligned}
$$

with the initial conditions

$$
\sum_{n=0}^{m-1} c_{i n} u^{(n)}(0)=\lambda_{i}, \quad i=0,1, \ldots, m-1 .
$$

Here, $a$ and $b_{n}(n=0,1, \ldots, m-1)$ are given analytical functions, $m-1<\theta \leq m, 0<\gamma_{0}<\gamma_{1}<\cdots<\gamma_{m-1}<\theta$ and $\mu, p_{n}, c_{i n}, \lambda_{i}$ denote given constants with $0<p_{n}<1(n=$ $0,1, \ldots, m)$. By using the shifted Jacobi-Gauss collocation method [39], we can approximate the fractional neutral functional-differential equations with proportional delays, without any artificial boundary and variable transformation. Let us first introduce some basic notation that will be used in the sequel.

Now we introduce the Jacobi-Gauss-Lobatto quadratures in two different intervals $(-1,1)$, and $(0, L)$. Denoting by

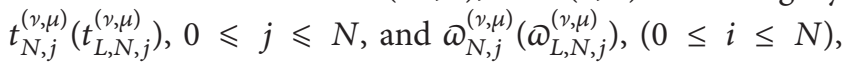
the nodes and Christoffel numbers of the standard (shifted) Jacobi-Gauss-Lobatto quadratures on $(-1,1),(0, L)$, respectively. Therefore, we can deduce that

$$
\begin{gathered}
t_{L, N, j}^{(\nu, \mu)}=\frac{L}{2}\left(t_{N, j}^{(\nu, \mu)}+1\right), \quad 0 \leq j \leq N, \\
\omega_{L, N, j}^{(\nu, \mu)}=\left(\frac{L}{2}\right)^{\nu+\mu+1} \omega_{N, j}^{(\nu, \mu)}, \quad 0 \leq j \leq N .
\end{gathered}
$$

Let $S_{N}(0, L)$ be the set of all polynomials of degree $\leq N$; then, for any $\phi \in S_{2 N+1}(0, L)$, we have

$$
\begin{aligned}
& \int_{0}^{L} w_{L}^{(v, \mu)}(t) \phi(t) d t \\
& \quad=\left(\frac{L}{2}\right)^{\nu+\mu+1} \int_{-1}^{1}(1-t)^{\nu}(1+t)^{\mu} \phi\left(\frac{L}{2}(t+1)\right) d t \\
& =\left(\frac{L}{2}\right)^{\nu+\mu+1} \sum_{j=0}^{N} \omega_{N, j}^{(v, \mu)} \phi\left(\frac{L}{2}\left(t_{N, j}^{(v, \mu)}+1\right)\right) \\
& =\sum_{j=0}^{N} \omega_{L, N, j}^{(\nu, \mu)} \phi\left(t_{L, N, j}^{(\nu, \mu)}\right) .
\end{aligned}
$$

We set

$$
S_{N}(0, L)=\operatorname{span}\left\{P_{L, 0}^{(v, \mu)}(t), P_{L, 1}^{(v, \mu)}(t), \ldots, P_{L, N}^{(v, \mu)}(t)\right\},
$$

and the inner product and norm are defined as

$$
\begin{gathered}
(u, v)_{w_{N}^{(\gamma, \mu)}}=\sum_{j=0}^{N} u\left(t_{L, N, j}^{(\nu, \mu)}\right) v\left(t_{L, N, j}^{(\nu, \mu)}\right) \omega_{L, N, j}^{(\nu, \mu)}, \\
\|u\|_{w_{N}^{(\gamma, \mu)}}=\sqrt{(u, u)_{w_{N}^{(\gamma, \mu)}}} .
\end{gathered}
$$

Obviously,

$$
(u, v)_{w_{N}^{(\nu, \mu)}}=(u, v)_{w^{(\nu, \mu)}}, \quad \forall u, v \in S_{2 N+1} .
$$

Thus, for any $u \in S_{N}(0, L)$, the norms $\|u\|_{w_{N}^{(\nu, \mu)}}$ and $\|u\|_{w_{N}^{(\nu, \mu)}}$ coincide.

Associating with this quadrature rule, we denote by $I_{N}^{P_{L}^{(p, \mu)}}$ the Jacobi-Gauss interpolation operator

$$
I_{N}^{P_{L}^{(\nu, \mu)}} u\left(t_{L, N, j}^{(\nu, \mu)}\right)=u\left(t_{L, N, j}^{(\nu, \mu)}\right), \quad 0 \leq k \leq N .
$$

The shifted Jacobi-Gauss collocation method for solving (22) and (23) is to seek $u_{N}(x) \in S_{N}(0, L)$, such that

$$
\begin{aligned}
D^{\theta}\left(u\left(t_{L, N-m, k}^{(\nu, \mu)}\right)+a\left(t_{L, N-m, k}^{(\nu, \mu)}\right) u\left(p_{m} t_{L, N-m, k}^{(\nu, \mu)}\right)\right) \\
=\mu u\left(t_{L, N-m, k}^{(v, \mu)}\right)+\sum_{n=0}^{m-1} b_{n}\left(t_{L, N-m, k}^{(v, \mu)}\right) D^{\gamma_{n}} u\left(p_{n} t_{L, N-m, k}^{(v, \mu)}\right) \\
+f\left(t_{L, N-m, k}^{(v, \mu)}\right), \quad k=0,1, \ldots, N-m, \\
\sum_{n=0}^{m-1} c_{i n} u^{(n)}(0)=\lambda_{i}, \quad i=0,1, \ldots, m-1 .
\end{aligned}
$$

We now derive the algorithm for solving (22) and (23). To do this, let

$$
u_{N}(t)=\sum_{h=0}^{N} a_{h} P_{L, h}^{(v, \mu)}(t), \quad \mathbf{a}=\left(a_{0}, a_{1}, \ldots, a_{N}\right)^{T} .
$$


We first approximate $D^{\theta} u(t)$ and $D^{\gamma_{n}} u(t), n=0,1, \ldots$, $m-1$, using (31). By substituting this approximation in (22), we get

$$
\begin{aligned}
D^{\theta} & \left(\sum_{h=0}^{N} a_{h} P_{L, h}^{(v, \mu)}(t)+a(t) \sum_{h=0}^{N} a_{h} P_{L, h}^{(v, \mu)}\left(p_{m} t\right)\right) \\
= & \mu \sum_{h=0}^{N} a_{h} P_{L, h}^{(v, \mu)}(t) \\
& +\sum_{n=0}^{m-1} \sum_{h=0}^{N} a_{h} b_{n}(t) D^{\gamma_{n}} P_{L, h}^{(v, \mu)}\left(p_{n} t\right)+f(t) .
\end{aligned}
$$

Making use of (16), we deduce that

$$
\begin{aligned}
& \sum_{h=0}^{N} \sum_{\sigma=0}^{N} a_{h} S_{\theta}(h, \sigma) P_{L, \sigma}^{(\nu, \mu)}(t) \\
& \quad+a(t) \sum_{h=0}^{N} \sum_{\sigma=0}^{N} a_{h} S_{\theta}(h, \sigma) P_{L, \sigma}^{(v, \mu)}\left(p_{m} t\right) \\
& =\mu \sum_{h=0}^{N} a_{h} P_{L, h}^{(\nu, \mu)}(t) \\
& \quad+\sum_{n=0}^{m-1} \sum_{h=0}^{N} \sum_{\sigma=0}^{N} a_{h} b_{n}(t) S_{\gamma_{n}}(h, \sigma) P_{L, \sigma}^{(\nu, \mu)}\left(p_{n} t\right)+f(t) .
\end{aligned}
$$

Also, by substituting (31) in (23) we obtain

$$
\sum_{n=0}^{m-1} \sum_{f=0}^{M} a_{i n} D^{(n)} P_{L, f}^{(\nu, \mu)}(0)=\lambda_{i}
$$

Now, we collocate $(33)$ at the $(N-m+1)$ shifted JacobiGauss interpolation points, yielding

$$
\begin{aligned}
& \sum_{h=0}^{N} \sum_{\sigma=0}^{N} a_{h} S_{\theta}(h, \sigma) P_{L, \sigma}^{(v, \mu)}\left(t_{L, N-m, k}^{(v, \mu)}\right)+a\left(t_{L, N-m, k}^{(\nu, \mu)}\right) \\
& \quad \times \sum_{h=0}^{N} \sum_{\sigma=0}^{N} a_{h} S_{\theta}(h, \sigma) P_{L, \sigma}^{(v, \mu)}\left(p_{m} t_{L, N-m, k}^{(v, \mu)}\right) \\
& =\mu \sum_{h=0}^{N} a_{h} P_{L, h}^{(v, \mu)}\left(t_{L, N-m, k}^{(\nu, \mu)}\right) \\
& +\sum_{n=0}^{m-1} \sum_{h=0}^{N} \sum_{\sigma=0}^{N} a_{h} b_{n}\left(t_{L, N-m, k}^{(v, \mu)}\right) S_{\gamma_{n}} \\
& +f\left(t_{L, N-m, k}^{(\nu, \mu)} \times(h, \sigma) P_{L, \sigma}^{(v, \mu)}\left(p_{n} t_{L, N-m, k}^{(\nu, \mu)}\right)\right.
\end{aligned}
$$

Next (34), after using (10), can be written as

$$
\sum_{n=0}^{m-1} \sum_{f=0}^{M}(-1)^{q} a_{i n} \frac{(-1)^{f-q} \Gamma(f+\mu+1)(f+\nu+\mu+1)_{q}}{L^{q} \Gamma(f-q+1) \Gamma(q+\mu+1)}=\lambda_{i} .
$$

TABLE 1: Absolute errors using SJGC method at $N=16$ for Example 3.

\begin{tabular}{lccc}
\hline$x$ & $v=\mu=-1 / 2$ & $\nu=\mu=0$ & $\nu=\mu=1 / 2$ \\
\hline 0.5 & $1.040 \cdot 10^{-3}$ & $2.799 \cdot 10^{-3}$ & $2.393 \cdot 10^{-4}$ \\
1.0 & $1.025 \cdot 10^{-3}$ & $4.986 \cdot 10^{-3}$ & $2.967 \cdot 10^{-4}$ \\
1.5 & $4.512 \cdot 10^{-3}$ & $4.006 \cdot 10^{-3}$ & $6.043 \cdot 10^{-4}$ \\
2.0 & $3.660 \cdot 10^{-3}$ & $2.487 \cdot 10^{-3}$ & $1.693 \cdot 10^{-4}$ \\
2.5 & $7.554 \cdot 10^{-3}$ & $3.817 \cdot 10^{-3}$ & $8.245 \cdot 10^{-4}$ \\
3.0 & $2.356 \cdot 10^{-3}$ & $2.975 \cdot 10^{-3}$ & $5.338 \cdot 10^{-4}$ \\
3.5 & $8.775 \cdot 10^{-3}$ & $8.662 \cdot 10^{-3}$ & $6.166 \cdot 10^{-4}$ \\
4.0 & $4.706 \cdot 10^{-3}$ & $5.449 \cdot 10^{-3}$ & $3.453 \cdot 10^{-4}$ \\
4.5 & $3.180 \cdot 10^{-3}$ & $2.261 \cdot 10^{-3}$ & $8.389 \cdot 10^{-5}$ \\
5.0 & $6.099 \cdot 10^{-3}$ & $1.002 \cdot 10^{-2}$ & $2.260 \cdot 10^{-3}$ \\
\hline
\end{tabular}

Finally, relations (35) and (36) generate $(N+1)$ set of algebraic equations which can be solved for the unknown coefficients $a_{j}, j=0,1,2, \ldots, N$, by using any standard solver technique.

\section{Numerical Results}

In this section, two fractional neutral functional-differential equations with proportional delays are solved by the SJGC method. We implement the method presented in this paper for these two examples to demonstrate the accuracy and capability of the proposed algorithm.

Example 3. Consider the following FNFDEs with proportional delay:

$$
\begin{array}{r}
u^{1 / 2}(t)=-u(t)+\frac{1}{4} u\left(\frac{t}{3}\right)+\frac{1}{3} u^{1 / 2}\left(\frac{t}{3}\right)+g(t) \\
u(0)=1, t \in[0,5]
\end{array}
$$

where

$$
\begin{aligned}
g(t)= & \frac{1}{\Gamma(1 / 2)} \int_{0}^{t}(t-s)^{-1 / 2} e^{s} d s \\
& +e^{t}-\frac{1}{4} e^{t / 3}-\frac{1}{3 \Gamma(1 / 2)} \int_{0}^{t}(t-s)^{-1 / 2} e^{s / 3} d s,
\end{aligned}
$$

and the exact solution is given by $u(t)=e^{t}$.

Table 1 lists the results obtained by the shifted Jacobi collocation method in terms of absolute errors at $N=16$ with $\nu=\mu=-1 / 2$ (first kind shifted Chebyshev collocation method), $\nu=\mu=0$ (shifted Legendre collocation method), and $\nu=\mu=1 / 2$ (second kind shifted Chebyshev collocation method). In the case of $\nu=1 / 2, \mu=-1 / 2$, the approximate solution by the presented method is shown in Figure 1, to make it easier to compare with the analytic solution. 


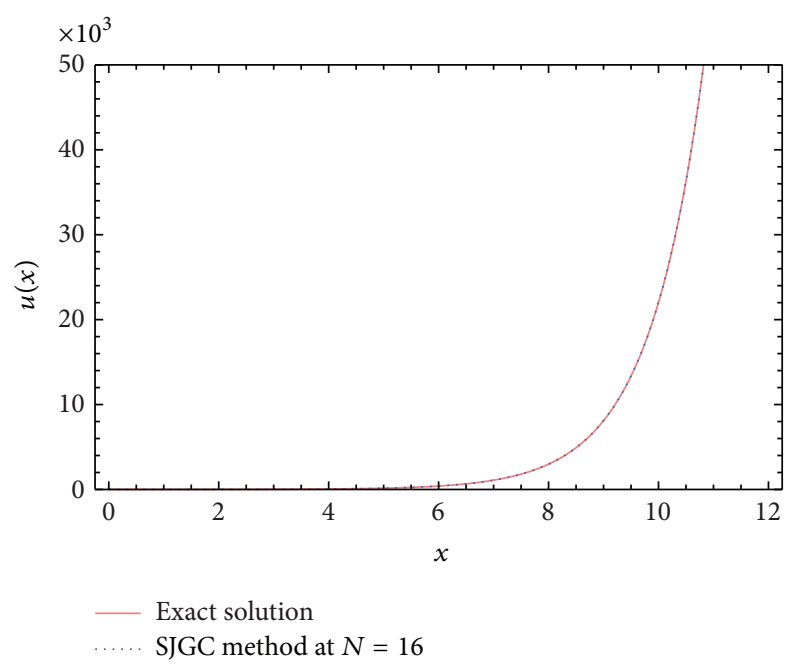

FIGURE 1: Graph of exact solution and approximate solution for $v=$ $1 / 2, \mu=-1 / 2$ at $N=16$ for Example 3 .

TABLE 2: Absolute errors using SJGC method at $N=16$ for Example 4.

\begin{tabular}{lccc}
\hline$x$ & $\nu=\mu=3 / 2$ & $\nu=\mu=0$ & $\nu=\mu=-1 / 2$ \\
\hline 0.1 & $1.462 \cdot 10^{-5}$ & $3.794 \cdot 10^{-5}$ & $2.092 \cdot 10^{-5}$ \\
0.2 & $7.095 \cdot 10^{-5}$ & $1.641 \cdot 10^{-4}$ & $9.636 \cdot 10^{-5}$ \\
0.3 & $1.717 \cdot 10^{-4}$ & $3.733 \cdot 10^{-4}$ & $1.947 \cdot 10^{-4}$ \\
0.4 & $3.355 \cdot 10^{-4}$ & $6.819 \cdot 10^{-4}$ & $3.394 \cdot 10^{-4}$ \\
0.5 & $5.649 \cdot 10^{-4}$ & $1.134 \cdot 10^{-3}$ & $6.780 \cdot 10^{-4}$ \\
0.6 & $8.468 \cdot 10^{-4}$ & $1.705 \cdot 10^{-3}$ & $1.203 \cdot 10^{-3}$ \\
0.7 & $1.183 \cdot 10^{-3}$ & $2.411 \cdot 10^{-3}$ & $1.650 \cdot 10^{-3}$ \\
0.8 & $1.592 \cdot 10^{-3}$ & $3.201 \cdot 10^{-3}$ & $1.934 \cdot 10^{-3}$ \\
0.9 & $2.083 \cdot 10^{-3}$ & $4.128 \cdot 10^{-3}$ & $2.295 \cdot 10^{-3}$ \\
1.0 & $2.681 \cdot 10^{-3}$ & $1.790 \cdot 10^{-3}$ & $2.903 \cdot 10^{-3}$ \\
\hline
\end{tabular}

Example 4. Consider the following FNFDEs with proportional delay:

$$
\begin{aligned}
u^{5 / 2}(t)= & u(t)+u^{1 / 2}\left(\frac{t}{2}\right)+u^{3 / 2}\left(\frac{t}{3}\right) \\
& +\frac{1}{2} u^{5 / 2}\left(\frac{t}{4}\right)+\frac{\Gamma(5)}{\Gamma(5 / 2)} t^{3 / 2}-\frac{\Gamma(4)}{\Gamma(3 / 2)} t^{1 / 2}-x^{4} \\
& +t^{3}-\frac{\Gamma(5)}{\Gamma(9 / 2)}\left(\frac{t}{2}\right)^{7 / 2}+\frac{\Gamma(4)}{\Gamma(7 / 2)}\left(\frac{t}{2}\right)^{5 / 2} \\
& -\frac{\Gamma(5)}{\Gamma(7 / 2)}\left(\frac{t}{3}\right)^{5 / 2}+\frac{\Gamma(4)}{\Gamma(5 / 2)}\left(\frac{t}{3}\right)^{3 / 2} \\
& -\frac{\Gamma(5)}{2 \Gamma(5 / 2)}\left(\frac{t}{4}\right)^{3 / 2}+\frac{\Gamma(4)}{2 \Gamma(3 / 2)}\left(\frac{t}{4}\right)^{1 / 2}, \quad t \in[0,1],
\end{aligned}
$$

subject to

$$
u(0)=0, \quad u^{\prime}(0)=0, \quad u^{\prime \prime}(0)=0,
$$

with exact solution $u(t)=t^{4}-t^{3}$.

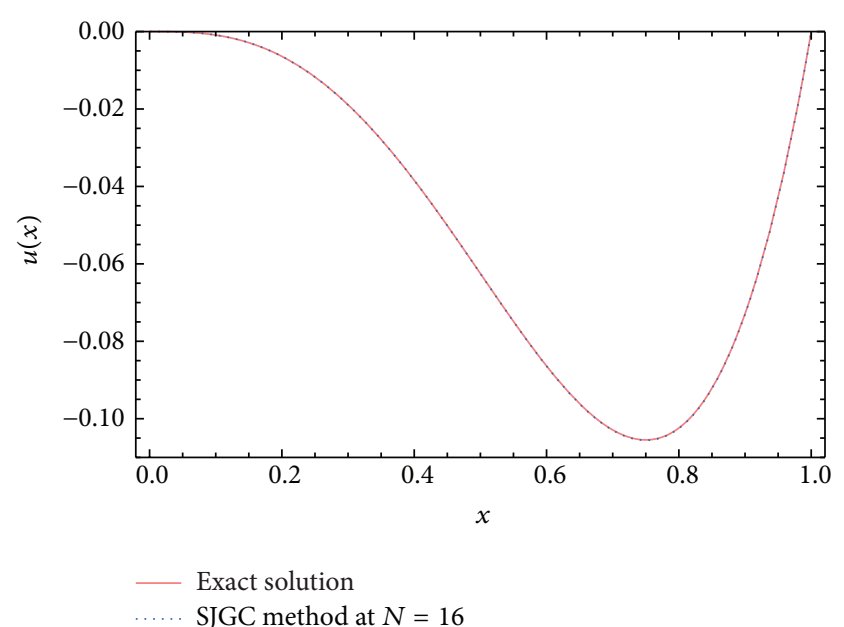

FIGURE 2: Graph of exact solution and approximate solution for $v=$ $\mu=1 / 2$ at $N=16$ for Example 4 .

In Table 2, we list the absolute errors obtained by the shifted Jacobi collocation method, with several values of $\nu, \mu$ and at $N=16$. It is clear that, for all Jacobi polynomials parameters, the results are stable. Meanwhile, Figure 2 presents the SJGC solution with $\nu=\mu=1 / 2$ at $N=16$ and exact solution, which are found to be in excellent agreement.

\section{Conclusion}

In this paper, we have proposed a numerical algorithm to solve a class of fractional delay differential equations. The Jacobi collocation approximation was developed to solve this problem. A number of collocation techniques can be obtained as special cases from the proposed technique. Numerical results were given to demonstrate the accuracy and applicability of the presented method.

\section{Conflict of Interests}

The authors declare that there is no conflict of interests regarding the publication of this paper.

\section{Acknowledgment}

This paper was funded by the Deanship of Scientific Research DSR, King Abdulaziz University, Jeddah. The authors, therefore, acknowledge and express their thanks to DSR technical and financial support.

\section{References}

[1] F. Flandoli and C. A. Tudor, "Brownian and fractional Brownian stochastic currents via Malliavin calculus," Journal of Functional Analysis, vol. 258, no. 1, pp. 279-306, 2010.

[2] R. L. Magin, C. Ingo, L. Colon-Perez, W. Triplett, and T. H. Mareci, "Characterization of anomalous diffusion in porous biological tissues using fractional order derivatives and 
entropy," Microporous and Mesoporous Materials, vol. 178, pp. 39-43, 2013.

[3] A. H. Bhrawy and M. A. Alghamdi, "A shifted Jacobi-GaussLobatto collocation method for solving nonlinear fractional Langevin equation involving two fractional orders in different intervals," Boundary Value Problems, vol. 2012, article 62, 2012.

[4] M. D. Choudhury, S. Chandra, S. Nag, S. Das, and S. Tarafdar, "Forced spreading and rheology of starch gel: viscoelastic modeling with fractional calculus," Colloids and Surfaces A, vol. 407, pp. 64-70, 2012.

[5] A. Atangana and N. Bildik, "The use of fractional order derivative to predict the groundwater flow," Mathematical Problems in Engineering, vol. 2013, Article ID 543026, 9 pages, 2013.

[6] A. Ghomashi, S. Salahshour, and A. Hakimzadeh, "Approximating solutions of fully fuzzy linear systems: a financial case study," Journal of Intelligent and Fuzzy Systems, vol. 26, pp. 367-378, 2014.

[7] F. Gao, X.-J. Lee, H. Tong, F. Fei, and H. Zhao, "Identification of unknown parameters and orders via cuckoo search oriented statistically by differential evolution for noncommensurate fractional-order chaotic systems," Abstract and Applied Analysis, vol. 2013, Article ID 382834, 19 pages, 2013.

[8] F. Gao, X. Lee, F. Fei, H. Tong, Y. Deng, and H. Zhao, "Identification time-delayed fractional order chaos with functional extrema model via differential evolution," Expert Systems with Applications, vol. 41, pp. 1601-1608, 2014.

[9] J. Deng and L. Ma, "Existence and uniqueness of solutions of initial value problems for nonlinear fractional differential equations," Applied Mathematics Letters, vol. 23, no. 6, pp. 676680, 2010.

[10] Y. Zhao, D.-F. Cheng, and X.-J. Yang, "Approximation solutions for local fractional Schrödinger equation in the onedimensional Cantorian system," Advances in Mathematical Physics, vol. 2013, Article ID 291386, 5 pages, 2013.

[11] Y. Zhao, D. Baleanu, C. Cattani, D.-F. Cheng, and X.-J. Yang, "Maxwell's equations on Cantor sets: a local fractional approach," Advances in High Energy Physics, vol. 2013, Article ID 686371, 6 pages, 2013.

[12] A.-M. Yang, Y.-Z. Zhang, C. Cattani et al., "Application of local fractional series expansion method to solve Klein-Gordon equations on Cantor sets," Abstract and Applied Analysis, vol. 2014, Article ID 372741, 6 pages, 2014.

[13] B. Jacobs and C. Harley, "Two hybrid methods for solving twodimensional linear time-fractional partial differential equations," Abstract and Applied Analysis. In press.

[14] Y. Z. Zhang, A. M. Yang, and X.-J. Yang, "1-D heat conduction in a fractal medium: a solution by the local fractional Fourier series method," Thermal Science, vol. 17, no. 3, pp. 953-956, 2013.

[15] A. Atangana and S. B. Belhaouari, "Solving partial differential equation with space- and time-fractional derivatives via homotopy decomposition method," Mathematical Problems in Engineering, vol. 2013, Article ID 318590, 9 pages, 2013.

[16] M. P. Tripathi, V. K. Baranwal, R. K. Pandey, and O. P. Singh, "A new numerical algorithm to solve fractional differential equations based on operational matrix of generalized hat functions," Communications in Nonlinear Science and Numerical Simulation, vol. 18, no. 6, pp. 1327-1340, 2013.

[17] A. H. Bhrawy and M. M. Al-Shomrani, "A shifted Legendre spectral method for fractional-order multi-point boundary value problems," Advances in Difference Equations, vol. 2012, article 8, 2012.
[18] Ş. Yüzbaşı, "Numerical solutions of fractional Riccati type differential equations by means of the Bernstein polynomials," Applied Mathematics and Computation, vol. 219, no. 11, pp. 6328-6343, 2013.

[19] A. Pedas and E. Tamme, "Numerical solution of nonlinear fractional differential equations by spline collocation methods," Journal of Computational and Applied Mathematics, vol. 255, pp. 216-230, 2014.

[20] A. H. Bhrawy, "A new numerical algorithm for solving a class of fractional advection-dispersion equation with variable coefficients using Jacobi polynomials," Abstract and Applied Analysis, vol. 2013, Article ID 954983, 9 pages, 2013.

[21] D. Baleanu, A. H. Bhrawy, and T. M. Taha, "Two efficient generalized Laguerre spectral algorithms for fractional initial value problems," Abstract and Applied Analysis, vol. 2013, Article ID 546502, 10 pages, 2013.

[22] X. Ma and C. Huang, "Spectral collocation method for linear fractional integro-differential equations," Applied Mathematical Modelling, vol. 38, no. 4, pp. 1434-1448, 2014.

[23] Y. Yang and Y. Huang, "Spectral-collocation methods for fractional pantograph delay-integrodifferential equations," Advances in Mathematical Physics, vol. 2013, Article ID 821327, 14 pages, 2013.

[24] F. Yin, J. Song, Y. Wu, and L. Zhang, "Numerical solution of the fractional partial differential equations by the twodimensional fractional-order Legendre functions," Abstract and Applied Analysis, vol. 2013, Article ID 562140, 13 pages, 2013.

[25] F. Yin, J. Song, H. Leng, and F. Lu, "Couple of the variational iteration method and fractional-order legendre functions method for fractional differential equations," The Scientific World Journal, vol. 2014, Article ID 928765, 9 pages, 2014.

[26] M. Kamrani, "Numerical solution of stochastic fractional differential equations," Numerical Algorithms, 2014.

[27] E. H. Doha, A. H. Bhrawy, D. Baleanu, and R. M. Hafez, "A new Jacobi rational-Gauss collocation method for numerical solution of generalized pantograph equations," Applied Numerical Mathematics, vol. 77, pp. 43-54, 2014.

[28] E. H. Doha, A. H. Bhrawy, M. A. Abdelkawy, and R. A. van Gorder, "Jacobi-Gauss-Lobatto collocation method for the numerical solution of $1+1$ nonlinear Schrödinger equations," Journal of Computational Physics, vol. 261, pp. 244-255, 2014.

[29] M. Tatari and M. Haghighi, "A generalized Laguerre-Legendre spectral collocation method for solving initial-boundary value problems," Applied Mathematical Modelling, vol. 38, no. 4, pp. 1351-1364, 2014.

[30] W. Shao and X. Wu, "The numerical solution of the nonlinear Klein-Gordon and Sine-Gordon equations using the Chebyshev tau meshless method," Computer Physics Communications, vol. 185, no. 5, pp. 1399-1409, 2014.

[31] E. H. Doha and A. H. Bhrawy, "An efficient direct solver for multidimensional elliptic Robin boundary value problems using a Legendre spectral-Galerkin method," Computers and Mathematics with Applications, vol. 64, no. 4, pp. 558-571, 2012.

[32] E. H. Doha, A. H. Bhrawy, and R. M. Hafez, "On shifted Jacobi spectral method for high-order multi-point boundary value problems," Communications in Nonlinear Science and Numerical Simulation, vol. 17, no. 10, pp. 3802-3810, 2012.

[33] A. H. Bhrawy, E. Tohidi, and F. Soleymani, "A new Bernoulli matrix method for solving high-order linear and nonlinear Fredholm integro-differential equations with piecewise intervals," Applied Mathematics and Computation, vol. 219, no. 2, pp. 482-497, 2012. 
[34] A. H. Bhrawy, M. M. Alghamdi, and T. M. Taha, "A new modified generalized Laguerre operational matrix of fractional integration for solving fractional differential equations on the half line," Advances in Difference Equations, vol. 2012, article 179, 2012.

[35] E. Ishiwata and Y. Muroya, "Rational approximation method for delay differential equations with proportional delay," Applied Mathematics and Computation, vol. 187, no. 2, pp. 741-747, 2007.

[36] X. Chen and L. Wang, "The variational iteration method for solving a neutral functional-differential equation with proportional delays," Computers and Mathematics with Applications, vol. 59, no. 8, pp. 2696-2702, 2010.

[37] M. Heydari, G. B. Loghmani, and S. M. Hosseini, "Operational matrices of Chebyshev cardinal functions and their application for solving delay differential equations arising in electrodynamics with error estimation," Applied Mathematical Modelling, vol. 37, no. 14-15, pp. 7789-7809, 2013.

[38] I. Podlubny, Fractional Differential Equations, vol. 198 of Mathematics in Science and Engineering, Academic Press, San Diego, Calif, USA, 1999.

[39] A. H. Bhrawy, "A Jacobi-Gauss-Lobatto collocation method for solving generalized Fitzhugh-Nagumo equation with timedependent coefficients," Applied Mathematics and Computation, vol. 222, pp. 255-264, 2013. 


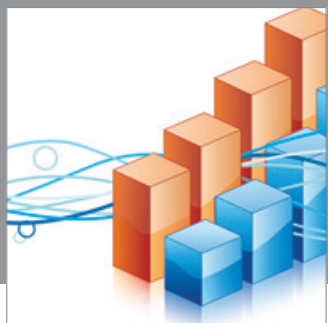

Advances in

Operations Research

mansans

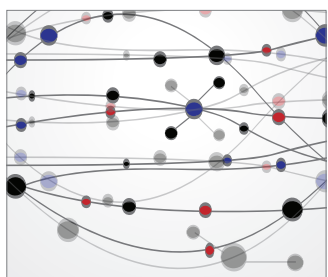

The Scientific World Journal
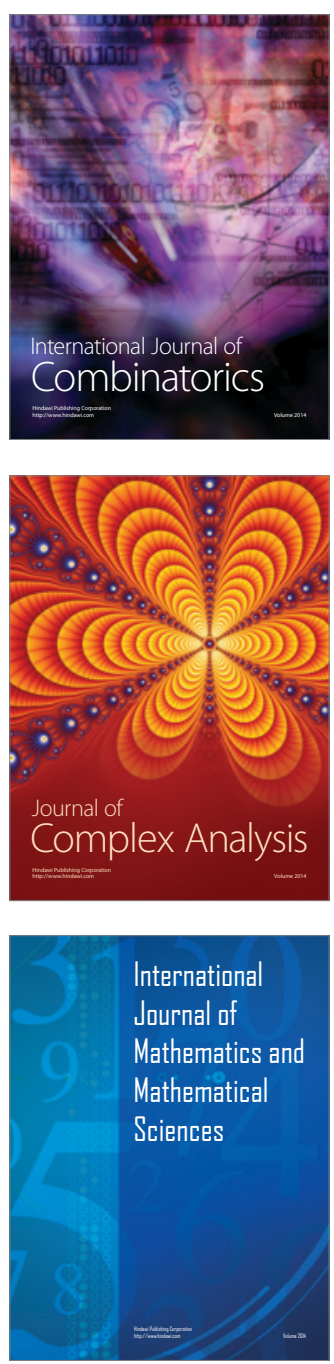
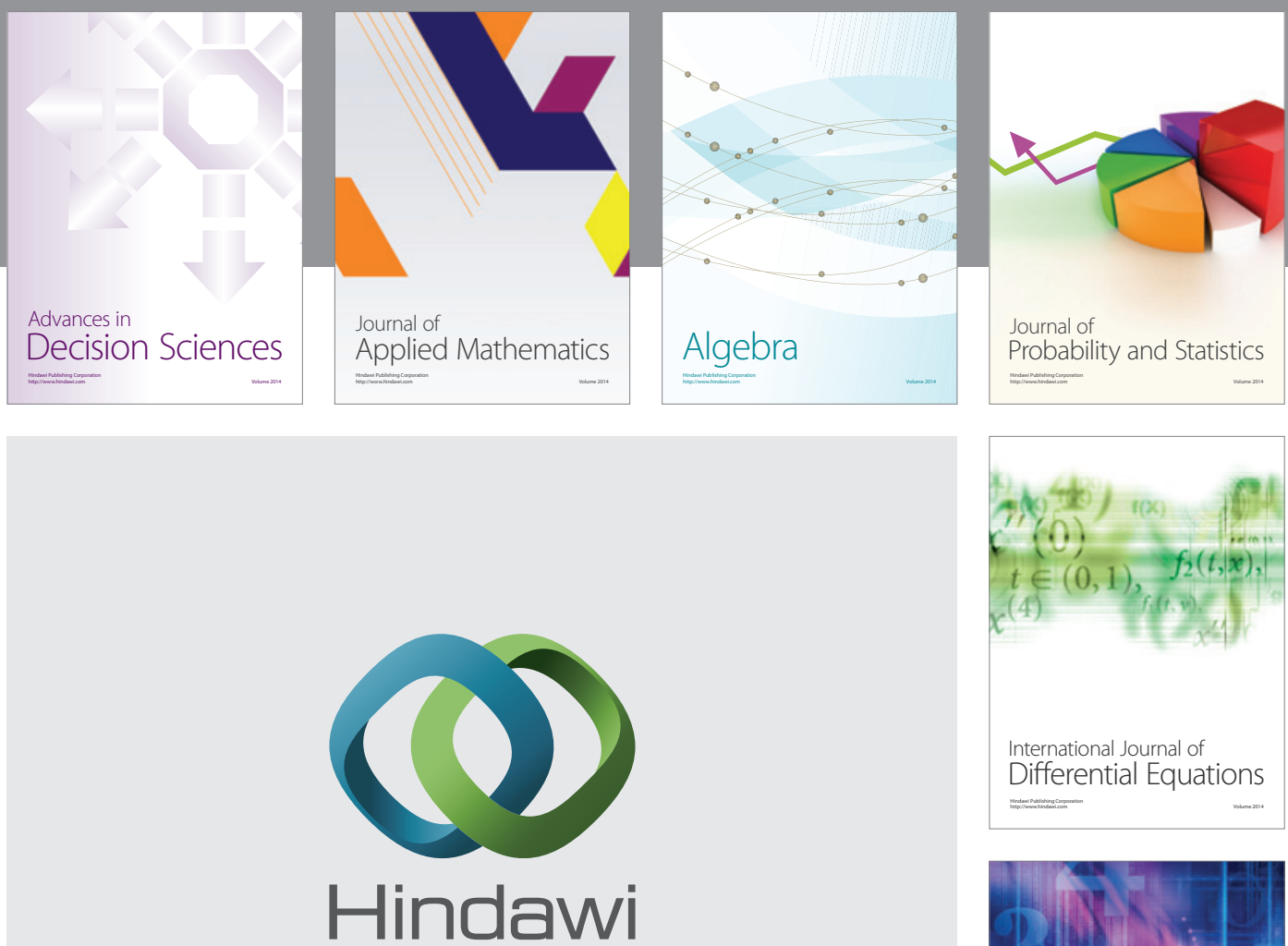

Submit your manuscripts at http://www.hindawi.com
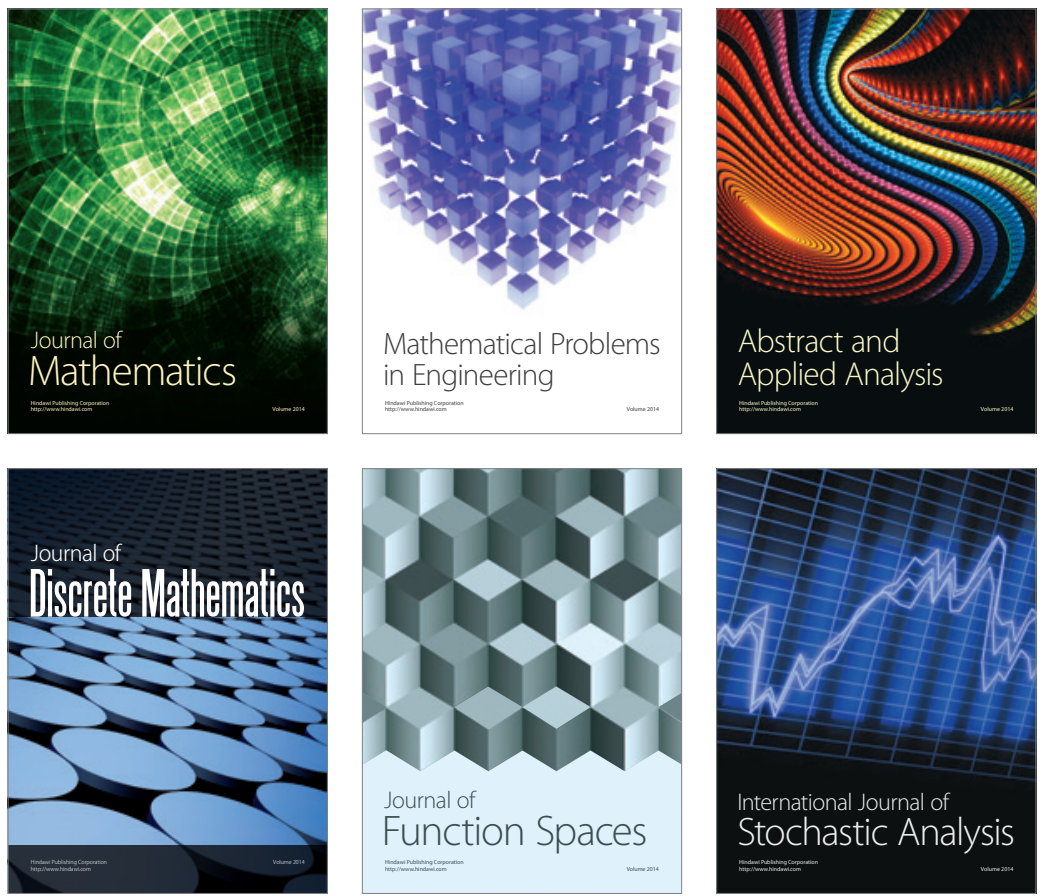

Journal of

Function Spaces

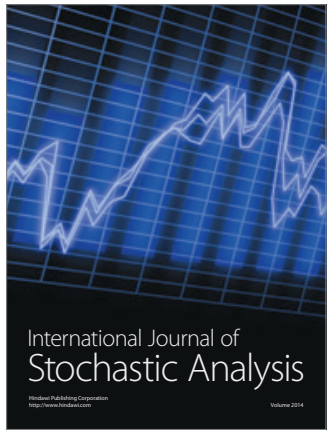

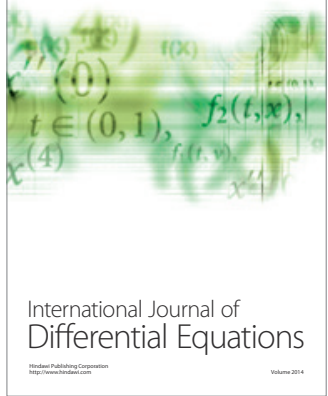
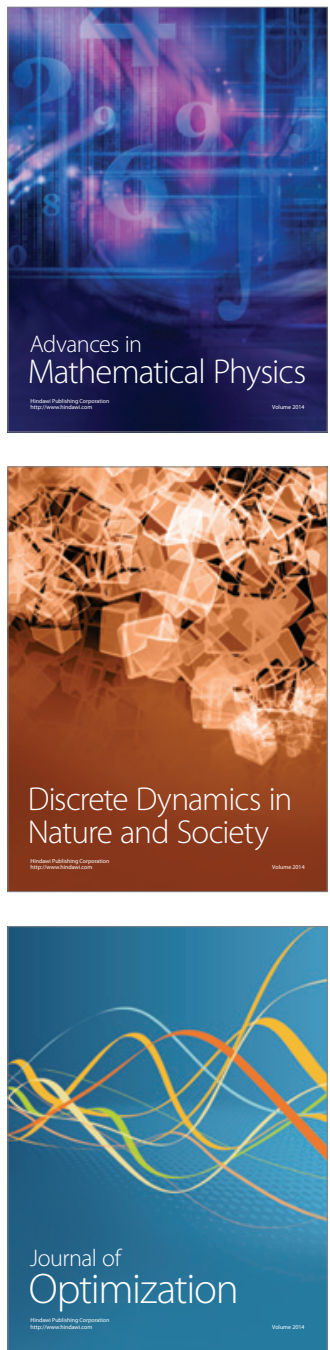